\title{
High-Speed Imaging and Spectro-Polarimetry with Subaru Telescope
}

\author{
Kazuhiro Sekiguchi \\ Subaru Telescope, 650 North A'ohoku Place, Hilo, Hawaii 96720 U.S.A.
}

Phil Charles

University of Southampton, Highfield, Southampton, S017 1BJ, UK

Liza van Zyl

Keele University, Keele, Staffordshire, ST5 5BG, UK

Guy F. Woodhouse

Rutherford Appleton Laboratory, Chilton Didcot, Oxfordshire, UK

\begin{abstract}
We are developing a high-speed option for the Faint Object Camera and Spectrograph (FOCAS) at the Cassegrain focus of the Subaru 8.2 meter telescope at Mauna Kea, Hawaii. FOCAS has been used for direct imaging, low-dispersion multi-object spectroscopy, imaging polarimetry and spectro-polarimetry of faint objects. The high-speed option utilizes an EEV CCD47-20 with the frame transfer operation to achieve up to $\sim 0.1 \mathrm{sec}$ time resolution. This paper presents a summary of the expected capability and the implementation schedule.
\end{abstract}

\section{Introduction}

The Faint Object Camera and Spectrograph (FOCAS) of the Subaru Telescope (Kashikawa et al. 2000) is a Cassegrain optical instrument, which is used for direct imaging, long-slit spectroscopy, multi-slit spectroscopy, and polarimetric imaging and spectropolarimetry. The updated characteristics of the FOCAS can be obtained from its web site.

http://www.naoj.org/Observing/Instruments/FOCAS/index.html

The combination of a large photon collecting power of Subaru Telescope and the FOCAS instrument would offer great opportunities to study MCVs in detail. However, the CCD camera currently used for the FOCAS has a relatively long read out time ( 90 seconds to read out $2048 \times 4096$ pixels). Therefore, it is not efficient for use on the high time resolution studies. In order to use the FOCAS for studies such as 1) studies of the accretion spots on accreting magnetic white dwarfs, 2) the structure of the mass transfer stream in MCVs, and 3) the nature 
of the QPOs $(0.1-10 \mathrm{~Hz})$ in accretion columns, we developed a high-speed CCD camera which can be used on the FOCAS, as to replace the standard CCD camera. We summarize the main characteristics of this new CCD camera and its implementation plan.

\section{High-Speed CCD Camera}

A high-speed CCD camera consists of a $1 \mathrm{~K} \times 1 \mathrm{~K}$ EEV CCD47-20 with 13.5 $\mu m$ pixel size. It covers $1.5^{\prime} \times 1.5^{\prime} \mathrm{FOV}$, and the pixel scale is 0.0935 arc$\mathrm{sec} / \mathrm{pix}$. The control electronics consists of a PC running Linux and the SDSU II CCD controller. The CCD is Peltier-cooled to approx -30 Celsius, yielding typical dark count rates of $\sim 0.3 \mathrm{e} / \mathrm{pix} / \mathrm{sec}$. Dark count can be ignored for short exposures in frame transfer mode $(1-30 \mathrm{~s})$. There are two gain settings; i) 1 e/ADU with a read noise of $<4 \mathrm{e}$ and ii) 2 e/ADU with a read noise of $<7$ e. The Analog-Digital Converter (ADC) saturates at 64,000 ADU and the CCD saturates, depending on the gain chosen, at $\sim 60,000$ e and $\sim 120,000$ e. The minimum read-out time (in frame transfer mode) is 40 millisec. The minimum exposure times in High Speed Photometry mode, is slightly longer than this.

Table 1. CCD Properties

\begin{tabular}{cl}
\hline No of pixels & $1,024 \times 1,024(\mathrm{EEV}$ CCD $47-20)$ \\
Pixcel size & $13.5 \mu \mathrm{m}$ \\
Pixel scale & $0.0935 \mathrm{arcsec} / \mathrm{pix}$ \\
FOV & $1.5 \times 1.5$ \\
Dark current & $\sim 0.3 \mathrm{e} / \mathrm{pix} / \mathrm{sec}$ \\
Gain & $1 \mathrm{e} / \mathrm{ADU}$ \\
& $2 \mathrm{e} / \mathrm{ADU}$ \\
Readout noise & $<4 \mathrm{e}$ with $1 \mathrm{e} / \mathrm{ADU}(<7 \mathrm{e}$ with $2 \mathrm{e} / \mathrm{ADU})$ \\
ADC Max. & $64,000(16 \mathrm{bit})$ \\
Saturation level & $\sim 60,000 \mathrm{e}(1 \mathrm{e} / \mathrm{ADU})$ and $\sim 120,000 \mathrm{e}(2 \mathrm{e} / \mathrm{ADU})$ \\
Min. exposure time & $\sim 0.1 \mathrm{sec}$.
\end{tabular}

\section{Implementation Schedule}

At this time (December 2002) the high-speed CCD camera is in the final testing state at the Rutherford Appleton Laboratory, U.K. It is expected to be shipped to the Subaru Telescope, Hilo, Hawaii by the end of March 2003. The first light of the high-speed CCD camera on the FOCAS and on the Subaru Telescope will be scheduled for the summer of 2003. After the initial performance verification tests, the high-speed option of the FOCAS will be available for the open use.

\section{References}

Kashikawa, N., Inata, M., Iye, M. (+ 16 co-authors), 2000, in SPIE Proc., 4008, 104 